\title{
Multiple Simultaneous Acute Stresses in Mice: Single or Repeated Induction
}

Rachael E. Hokenson ${ }^{1}$, Mikko Oijala ${ }^{2}$, Annabel K. Short ${ }^{3}$, Jessica L. Bolton ${ }^{3}$, Yuncai Chen ${ }^{3}$, Jenny

Molet $^{3}$, Pamela M. Maras ${ }^{3}$, Tallie Z. Baram ${ }^{1,3,4, *}$ and Gyorgy Lur ${ }^{2, *}$

${ }^{1}$ Department of Anatomy and Neurobiology, University of California-Irvine, Irvine, CA, USA; ${ }^{2}$ Department of Neurobiology and Behavior, University of California-Irvine, Irvine, CA, USA; ${ }^{3}$ Department of Pediatrics, University of California-Irvine, Irvine, CA, USA; ${ }^{4}$ Department of Neurology, University of California-Irvine, Irvine, CA, USA

*For correspondence: tallie@uci.edu; glur@uci.edu

[Abstract] Stress is crucial to the survival of an organism, but excessive stress can lead to psychological disorders including depression, anxiety, substance abuse, and suicidality. The prevailing notion is that chronic stress promotes adverse outcomes on brain and body health, whereas acute stressors are generally benign. Notably, acute events such mass shootings or natural disasters are now emerging as significant sources of cognitive and emotional problems including post-traumatic stress disorder (PTSD). These events are characterized by the simultaneous occurrence of physical, emotional, and social stresses, which last minutes to hours. Hence, there is a need to model such multiple concurrent acute stresses (MAS) to uncover the mechanisms by which they lead to profound adverse outcomes. The MAS paradigm described here involves simultaneously exposing a rodent to several different stressors including restraint, crowding, and jostling alongside peers in a brightly lit and very noisy environment. Moreover, the MAS paradigm can be used once or imposed repeatedly to emulate complex, repeated modern life stresses, advancing our mechanistic understanding of consequent mental and cognitive impairments.

Keywords: Acute stress, Chronic stress, Multimodal stress, Mouse, Restraint, Memory, Neuroscience

[Background] Stress is common and inevitable. Severe or chronic stress can result in an array of cognitive, emotional, and physical problems. To understand the molecular, cellular, and physiological underpinnings of stress and its influence on brain function, stress must be modeled in the laboratory. It is conventionally accepted that chronic stress leads to adverse health outcomes while acute stress can be benign or advantageous. For example, chronic stress induced by unpredictable intermittent restraint impaired spatial memory (Peay et al., 2020), whereas one hour of acute restraint stress enhanced novel object recognition memory in male rats (Brivio et al., 2020). However, previous approaches to acute stress typically imposed a single or "simple" stress. Notably, acute stressful life events such as mass shootings or natural disasters may only last minutes to hours, yet consist of physical, emotional, and social stresses occurring at the same time. These events are now being shown to provoke negative long-term outcomes such as PTSD in a significant number of individuals (North et al., 1994; Lowe and Galea, 2017; Musazzi et al., 2017; Novotney, 2018). Therefore, there is an unmet need to model MAS in the laboratory to discover the mechanisms by which they promote negative outcomes. 
We have developed a multiple concurrent acute stresses (MAS) paradigm (Figure 1) to examine cognitive and "emotional" impairments resulting from these types of stress (Maras et al., 2014). MAS utilizes mild to moderate stressors: restraint, awareness of peer discomfort, bright lighting, loud noise, and jostling, but delivers these to the animal simultaneously within as little as one hour. MAS lasting two-hours has lasting impact on memory and hippocampal spine integrity, assessed via spatial memory tests, slice physiology, and several quantitative methods to determine spine density and loss (Maras et al., 2014; Chen et al., 2016). For example, adult male mice subjected to restraint only stress or noise only stress, paradigms that are more commonly used in labs to model acute stress, did not have these profound memory deficits. MAS activates additional and distinct brain networks compared to single acute stressors, promoting cross-correlated activation of amygdala and the bed nucleus of the stria terminalis (Maras et al., 2014). These results highlight that MAS may employ different mechanisms to impact brain function compared to commonly utilized acute stressors. Thus, MAS may provide a more optimal model for concurrent acute life stresses that produce profound, negative outcomes.

In contrast to simple acute stress, chronic stress may promote neuropsychiatric pathology. Several protocols have been developed to model chronic stress in rodents. Repeated physical restraint in a conical tube or similar restraining device is a popular paradigm (Kim and Han, 2006). To avoid potential habituation to a single stressor over an extensive period of time, the unpredictable chronic mild stress procedure [UCMS or CUMS, with variations including chronic unpredictable stress (CUS or UCS), chronic mild stress (CMS), and chronic variable stress (CVS)] subjects rodents to several weeks of various stressors, such as noise, restraint, bedding deprivation, and light cycle disruption (Willner, 1997 and 2017; Burstein and Doron, 2018). While this protocol is widely used, it is quite labor intensive and some strains of mice, including the commonly used C57BL/6 strain, can be resistant to the effects of UCMS unless the duration of the protocol is greatly extended (Monteiro et al., 2015).

To circumvent issues with some chronic stress protocols and also engage the same networks as those activated in a single exposure to MAS, the MAS paradigm has been adapted to chronic applications. Repeated multiple concurrent stressors (RMS) for one hour a day across 10 days in adolescent, male C57BL/6 mice induce the loss of sensory and retrosplenial cortical input to the posterior parietal cortex as well as disrupt working memory performance (Libovner et al., 2020). This extensive circuit disruption is not observed in mice subjected to the same amount of restraint only stress, highlighting the specific mechanisms engaged during exposure to MAS.

The MAS protocol facilitates the investigation of the consequences of stress that may be limited in duration, such as natural disasters or mass shootings. This paradigm can further be adapted to chronic applications, capitalizing on the network activation of a single exposure that may not be similarly engaged in other chronic stress paradigms. Furthermore, this protocol is technically simple and titratable, promoting robust and reproducible results. 


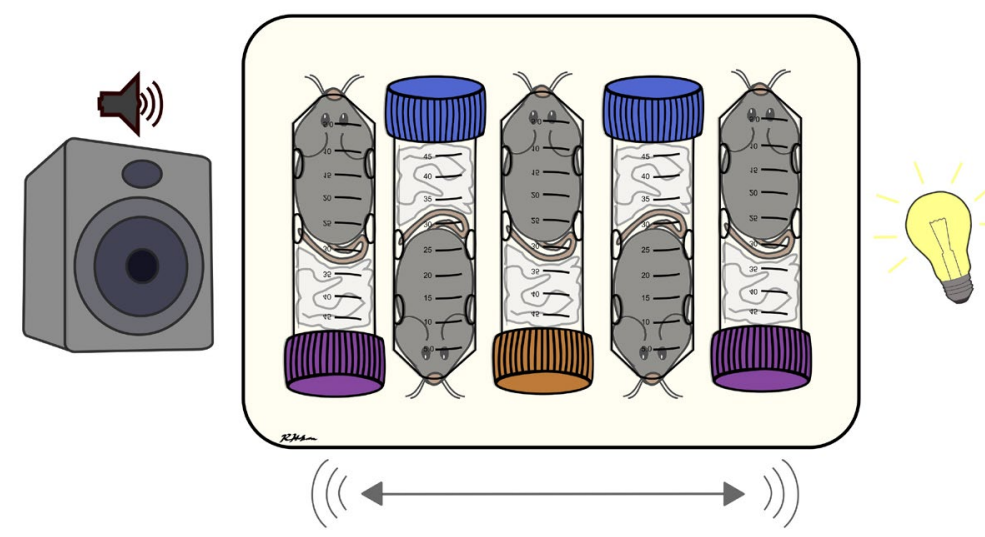

Figure 1. Multiple Concurrent Acute Stresses Paradigm. Mice are subjected to physical, social, and emotional stresses concurrently through restraint, crowding, jostling, loud noise (e.g., music or random, high frequency beeps), and bright lights.

\section{Materials and Reagents}

1. Restraint tubes: $50 \mathrm{ml}$ conical tubes (approximately $30 \mathrm{~mm}$ diameter $115 \mathrm{~mm}$ length) (Corning ${ }^{\circledR}$, catalog number: 430921, others) with openings to permit breathing (see Procedure)

2. Paper towels (Scott ${ }^{\circledR}$ Multi-Fold Towels, catalog number: 01804, others)

3. C57BL/6 adolescent or adult mice (see Notes on "Variations of the MAS protocol")

4. $95 \%$ ethanol for cleaning

\section{Equipment}

1. Multipurpose rotator/orbital shaker (Thermo Scientific, model: 2314) or benchtop laboratory rocker (IBI Scientific ROCAA115S Hi/Lo Profile Rocker, 115V, amazon.com)

2. Stereo system (Sony CFD-510 Radio Cassette-Corder with Mega Bass, others) or audio amplifier (e.g., Stereo 20W Class D Audio Amplifier, MAX9744, Adafruit product ID: 1752 but any audio amplifier will work), high frequency response speakers (e.g., High Performance Piezo Tweeters for Car Audio 1.5" 400 Watts 4 ohm Super High Frequency, amazon.com), and an Arduino UNO R3 board (Arduino, product code: 8058333490090) with 2 x $12 \mathrm{~V}$ power supply (12V 2A 24W AC DC Switching Power Supply Adapter, amazon.com)

3. Audio in format compatible with stereo or Adruino code (provided)

4. 1-2 empty mouse housing cages or plastic box that will fit on rotator $(20 \mathrm{~cm}$ width $\times 36 \mathrm{~cm}$ length $x 13 \mathrm{~cm}$ height or similar)

5. Scale with tenth of a gram precision (Sartorius, model: PT600, others)

6. Decibel meter (or equivalent cellular phone application)

7. Ear plugs $\left(3 \mathrm{M}^{\mathrm{TM}}\right.$, catalog number: 70005103141 , others)

8. C-Clamp or vise to hold conical tube

9. Power drill with bits (up to $1 / 4$ in.) 
10. Sandpaper

11. Jumper cables for Arduino wiring (EDGELEC 120pcs Breadboard Jumper Wires, amazon.com)

12. USB cable (USB Data Sync Cable for Arduino UNO/Arduino Mega 2560 Rev 3 R3 Microcontroller, amazon.com)

13. Power strip (AmazonBasics 6-Outlet Surge Protector Power Strip, amazon.com)

14. LED light source (e.g., RGB LED Spotlight, LOFTEK 10W, amazon.com)

\section{Procedure}

Note: Approval must be obtained from the appropriate animal use committees prior to conducting this experiment.

\section{Part I: Preparing Materials}

\section{A. Constructing restraint tubes}

1. Construct as many restraint tubes as you would need to stress your desired maximum number of mice at once plus several extras. If all mice are stressed within one single cage, maximum mice would be 6-8 (depending on cage size) or doubled if using two cages. Within a cage there should be enough empty space for the restrained mice to be jostled at least several centimeters. If using multiple cages these should be placed on the rotator side by side. This is not suggested if both cages together hang over the edges of the rotator substantially. Practice operating the rotator at the desired speed without mice to ensure the cages will not slip off and consider adding traction to the cage bottoms to prevent slipping.

Note: Two mice minimum should be used to include a component of "social stress" (see "Variations of the MAS protocol" in the Notes section). Stressing too many mice at once may have mixed results and will make setup (if only one experimenter is performing) more technically challenging. See "Behavioral Testing Considerations" in the Notes section for tips on staggering this procedure to accommodate more animals without compromising important time points.

2. Use $50 \mathrm{ml}$ conical plastic tubes of any brand with approximate dimensions of $30 \mathrm{~mm}$ diameter and $115 \mathrm{~mm}$ length. Width and length of tube should be generous enough that a mouse will be snug, neither squashed nor free enough to move around more than wriggling. We do not recommend using mice over $38 \mathrm{~g}$ with this tube size or else they may be injured upon removal. Give tubes unique identifiers (numbers, letters, and/or different colored lids) to better keep track of mice during stress.

3. Hold $50 \mathrm{ml}$ tube steady in a vice or clamp (Figure 2A). Drill an air-hole at the bottom point of the tube. Start with a small enough drill bit to make a clean hole then gradually increase bit size until the hole is properly sized. This hole should be just large enough for a mouse's nose to fit through $(\sim 6 \mathrm{~mm})$. Larger holes might cause the mouse to get their teeth stuck, which could result in injury. Make openings smooth and remove any jagged plastic with sandpaper. 
4. Repeat this process to make similar sized holes along the length of the tube (Figure 2B). There should be enough openings to provide adequate ventilation, but not enough that the mouse's limbs will frequently get caught. Five total openings is a good minimum. Position these holes closer to the conical tip of the tube (as opposed to the lid), as this is where the mouse will be located.

A

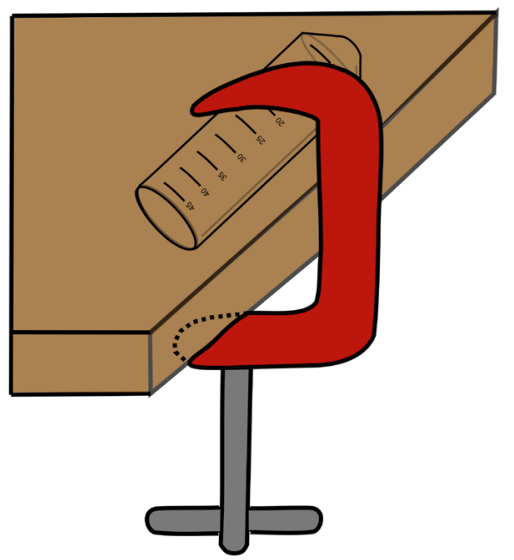

B

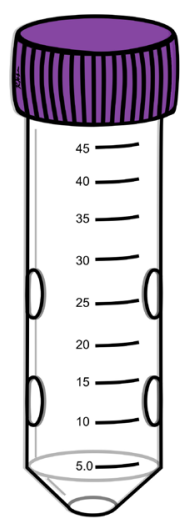

Figure 2. Constructing restraint tubes. A. Hold a conical tube steady with a C-clamp (as shown) or in any other clamp or vise while drilling. The tube should be clamped tight enough to not be moved but without being crushed. B. An example ventilated restraint tube. Drill one hole at the tip of the conical tube for the nose. Drill several holes along the sides of the restraint tube to add additional ventilation. Exact number of air holes can vary but avoid too many or too large of holes that will cause limbs to get stuck.

5. This completes one restraint tube. Repeat process for desired number of tubes.

6. Store restraint tubes with their lids in a dust-free bag or box until use.

B. Generating loud noise

Option 1: Utilize any available stereo system capable of playing music at a level of 90 decibels or higher. Typically rap or hip-hop music is played as described in Part II Step A4.

Option 2: Set up Arduino UNO with stereo amplifier and speaker.

1. Download and install the Arduino Interactive Development Environment (https://www.arduino.cc/en/Main/Donate).

2. Connect the Arduino Uno to a PC or Mac using the USB cable.

3. Run the peeper.ino file by double clicking. This will launch the Arduino IDE.

4. Click Upload in the Arduino IDE. From this point onwards there is no need for a PC; the Arduino Uno will run the noise generator when powered. 
5. Use the jumper cables to connect Arduino UNO Pin 13 and Ground to the MAX 9744 audio amplifier ("L" and "-" ports, respectively) and plug the speaker cables into the amplifier output (see Figure 3 for wiring diagram).

6. Place the speaker in the cage atop the laboratory shaker.

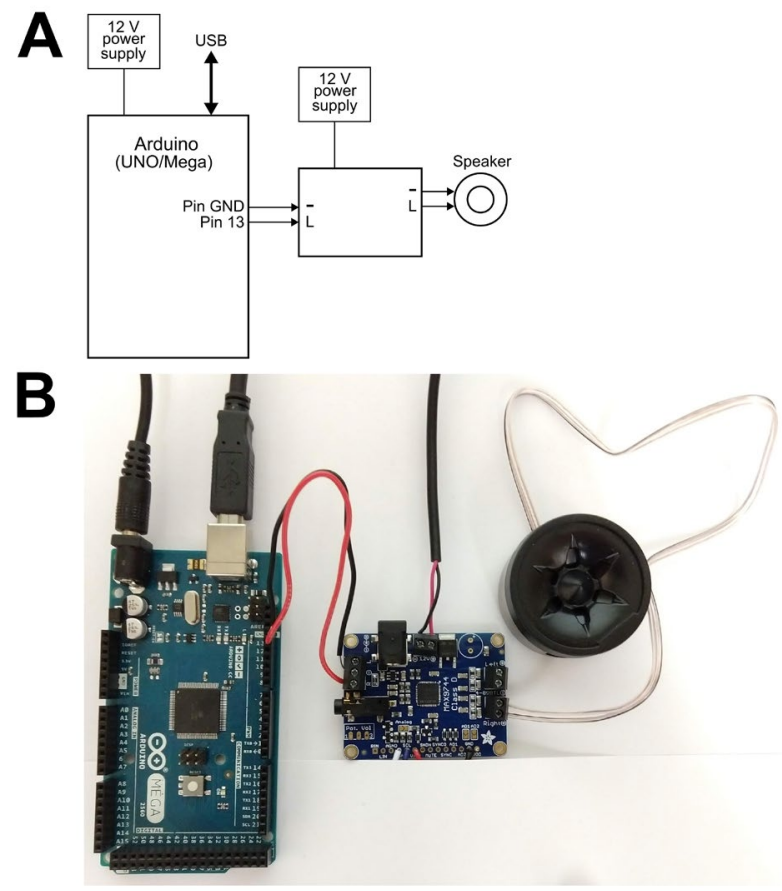

Figure 3. Wiring diagram for sound generator setup. A. The Arduino can be wired as represented schematically. Jumper cables can be directly soldered onto the MAX 9744 amplifier board or secured through the provided screw terminal. After assembly and sketch upload, the whole assembly can be started and ran for the period of the stress exposure by powering the two $12 \mathrm{~V}$ power supplies without the need to connect to a computer. B. An actual Arduino setup is displayed.

\section{Part II: Running MAS}

A. Setting up MAS

1. Clean restraint tubes before use. Rinse the inside and outside of the tube and cap with distilled water and scrub with your fingers or an appropriately sized bottle brush. Give extra attention to the air holes where grime may collect. Liberally rinse with $95 \%$ ethanol and leave upside down to dry. Clean tubes either the night before or several hours prior to stress. Restraint tubes must be completely dry before using.

2. Prepare paper towels to cushion the mouse in the tube. Tear paper towels in halves and quarters. For an average male mouse (25-38 g), a half paper towel is generally sufficient. For smaller mice and most females (15-25 g), a half plus a quarter paper towel may be needed to keep 
them sufficiently immobilized. Adjust preparations according to your average mouse size and paper towel thickness.

3. In the experiment room, set up the rotator with an empty cage or two sitting on top. Have the rotator set at the desired speed. For Thermo Scientific shaker Model No. 2314 this is the fourth setting from max, where max speed is 220 RPM (see Video 1 for speed demonstration).

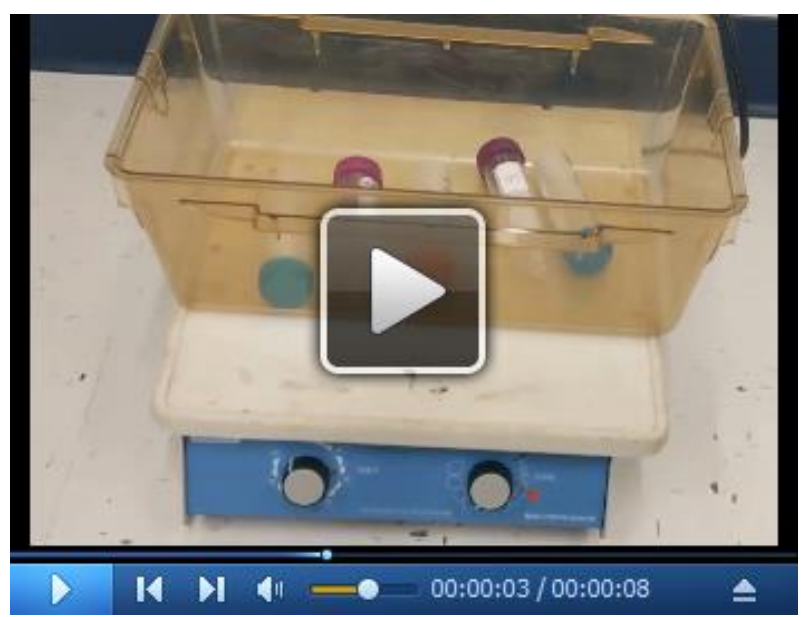

Video 1. MAS shaker speed. To create the "jostling" physical stressor, the Thermo Scientific shaker Model No 2314 is set to the fourth setting from max speed. The restraint tubes are empty in this demonstration.

4. Before running stress, test the audio volume. Music (typically rap or hip-hop) or noise can be played. In the Baram lab, "Silly Ho," from TLC's "Fan Mail" (1999) is played on loop (Maras et al., 2014; Chen et al., 2016). Insert ear plugs, turn on audio, and hold the decibel meter or phone with decibel meter app where the shaker will be located. Set volume such that the meter reads between 85-90 dB. This volume will likely fluctuate. Aim for an average reading between 85-90 dB. If using a traditional stereo system, consider marking this point on the volume knob with tape or marker for quick access in the future. Audio should be set to repeat on an infinite loop. Alternatively, in the Lur lab, noise is generated by an Arduino Uno and consists of $0.5-1 \mathrm{~s}$ long beeps randomly selected from a range of $15-30 \mathrm{kHz}$ at $0.5-3 \mathrm{~s}$ random intervals (Libovner et al., 2020) as described in Part I Section B Option 2.

5. Put each restraint tube on the counter next to its cap with a stack of pre-cut paper towels at hand. Also have a notebook handy for recording mouse tube assignments, pre and post MAS weights, and start and end times of MAS.

\section{B. Starting and running MAS}

1. Bring mice from their housing room to the experimental room either in their housing cage or in a transport cage. Do not bring control mice to this room while stress is running. Keep control mice in the housing room or transport to a different room. 
2. Designate a labeled tube to each mouse. This will allow you to identify mice during stress and make any necessary notes. Ear clip or tail markings will not be clear while the mouse is restrained. Record the order in which each mouse is or will be restrained.

3. Record the weight of each mouse before restraining.

4. A few minutes before the intended MAS start time, insert ear plugs then begin restraining mice. Exact time to begin restraining depends on your experience/speed, mouse cooperation, and how many mice need to be restrained. A well-trained experimenter with a handled mouse should take approximately $30 \mathrm{~s}$ to one minute to restrain each mouse.

5. When ready to restrain, grab and hold the mouse by the base of the tail, lifting them up so that their hind limbs are against the walls of a corner of the cage and their forelimbs and nose are against the floor or bedding. Hold a finger or two against the mouse's back to limit movement and help push the mouse into the tube. Bring the open end of the restraint tube to their nose. Insert the mouse's head into the tube and bring the tube up towards yourself while gently pushing with your finger(s) on their back to fully encase the mouse in the tube. The mouse's nose should be against or partly sticking out from the breathing hole at the base of the tube. The mouse should fit into the tube easily. If the mouse is too large, do not shove into the restraint tube. Instead make restraint tubes out of larger tubes if possible or restrain by a different method.

6. Keep one finger on the mouse's tail end to keep within the tube. Let the tail fall into the tube or gently push it to curl it inside the tube. Grab a half paper towel and push this into the space behind the mouse, making sure to completely cover the tail but not forcefully enough to hurt the mouse. If there is still a lot of space after the half paper towel, consider inserting another quarter towel.

7. Once the paper towel(s) are in place, carefully screw on the lid. Be very careful not to get the mouse's tail caught in the lid (Figure 4). This entire process should ideally take less than one minute to avoid any additional stress from experimenter manipulation. Mice that are accustomed to handling will be quicker and easier to restrain and should require less "stressful" handling. 


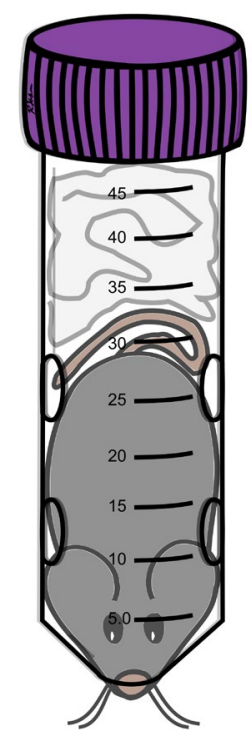

Figure 4. Restrained mouse. Nose should be against or partly sticking out of the nose hole. Paper towel(s) should fill the remaining space behind the mouse to keep the mouse from turning around. Mouse should be able to wriggle slightly. Whiskers may stick out of nose hole at some point during restraint.

Note: The mouse's tail should be mostly folded down before inserting the paper towel such that once the towel is in place the tail is not visible. This will prevent the tail from getting caught in the lid which can result in bleeding and potential fracturing of tail. If such an injury is found, monitor the mouse's health. Intervene if bleeding does not stop.

8. The restrained mouse can either be placed into the cage that is on the shaker or remain on the counter while the other mice are being restrained.

9. Repeat the restraint process for each mouse.

10. Once all mice are restrained, put the mice into the cage on the laboratory shaker. If possible, alternate mice so that each is next to a mouse who is not their cage mate to increase social stress.

11. Switch on laboratory shaker for continuous shaking and turn on audio. Start a timer (counting up) and record MAS start time (Figure 5). 


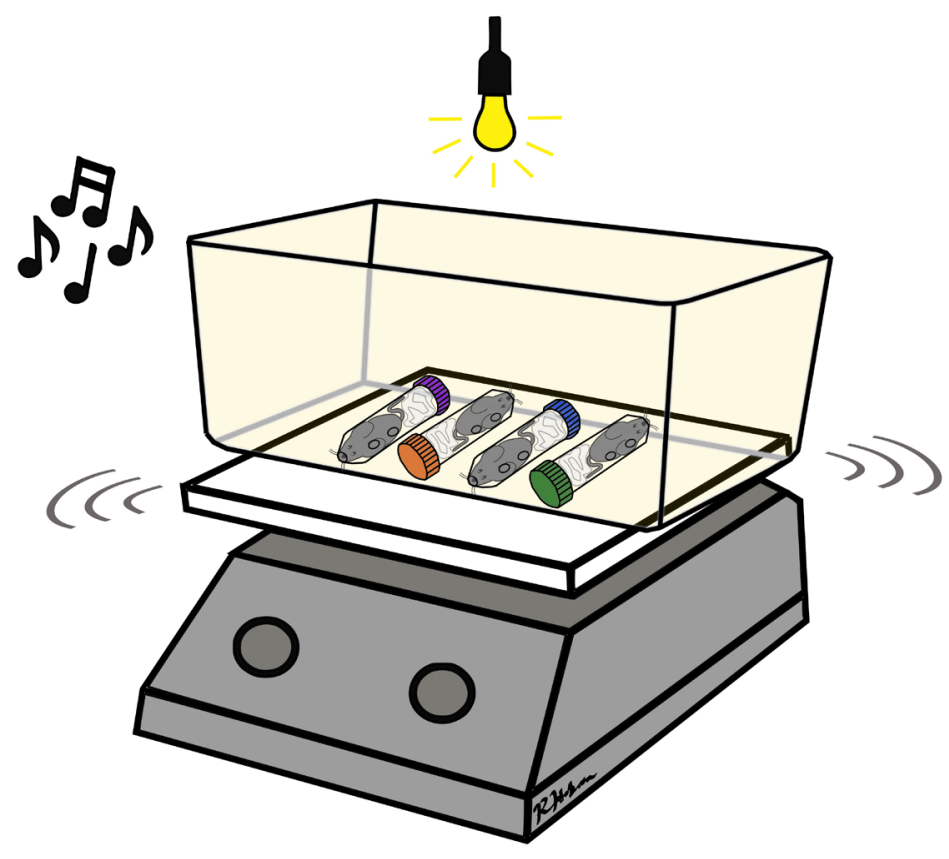

Figure 5. Mouse crowding and jostling. Restrained mice are arranged in alternating positions in a container on top of the laboratory shaker placed in a brightly lit room in the presence of 90 $\mathrm{dB}$ music or random tones.

12. Continue running MAS for the desired duration (e.g., one, two, or five hours).

13. All mice must be checked periodically during stress (every 10 or $15 \mathrm{~min}$ ) to ensure their health and safety. At each check point, pick up each tube one at a time. Ensure the mouse is still warm. Mice should be able to wriggle very slightly as you hold them still for this moment. Realign tubes in the center of the cage. The rotator should remain on during this period.

Note: If the mouse has turned their head or is in any position that may limit breathing or possibly cause injury, unscrew cap, remove paper towel, and adjust mouse before returning to the shaker. Mice will urinate and defecate while in the restraint tube.

14. During mouse checks, ensure that house lights in the room are staying on, especially in rooms where lights may be motion activated.

\section{Ending MAS and disassembling}

1. Turn off rotator and stereo system after the intended duration for MAS has elapsed. If desired, keep the timer counting to reference for later experiments.

2. Remove mice from the tubes in the same order in which they were inserted. Ideally your speed of removing mice should be similar to your speed of restraining mice, such that each mouse's total restraint time is equivalent.

3. To remove the mouse: unscrew the restraint tube cap and pull out paper towel(s) (feces will come with them). Grab the tail as close to the base as possible and gently tug. The mouse should slide out and may start backing out on their own. If the mouse's nose or teeth are stuck in the nose hole, lightly push nose with finger to set mouse free. Do not pull too forcefully or this 
will injure the mouse. Unless the mouse was too large to fit, the mouse should come out easily. Remove the mouse in one fluid motion or else they may burrow themselves further into the tube to escape from you. For a well-trained experimenter, removing a mouse should take no more than 30 s per mouse.

4. Weigh the mouse once removed from the tube. Mice generally lose a small amount of weight (1-4\% of body weight, usually a gram or less) during this period and will have a very rumpled appearance upon exiting the tube. Any dramatic weight loss may be due to dehydration and the mouse's health should be monitored. Mice may continue to look rumpled for several hours but should be properly groomed by the next day. The mouse's health should be monitored if they have not resumed normal grooming.

5. Return the mouse to the housing or transport cage.

6. Repeat process for all mice.

7. Depending on your experiment, mice can either be immediately sacrificed for analysis or brought back to the housing area or behavior suites to await later testing (see Notes on "Behavioral Testing Considerations").

8. Empty restraint tube of animal waste. Again, rinse the inside and outside of the tube and cap with distilled water and scrub with your fingers or an appropriately sized bottle brush. Give extra attention to the air holes where grime may collect. Liberally rinse with $95 \%$ ethanol and leave upside down to dry. Store tubes with lids in a dust-free bag or container. Dispose of tubes if they become damaged or cannot be cleaned.

This completes one session of MAS. To study the effects of chronic MAS, repeat the above procedure for the desired period, starting MAS at roughly the same time each day.

\section{Notes}

A. Variations of the MAS protocol

1. For some experiments you may have access to only a few mice and/or only a few mice will be available for testing at a given time. We have employed as few as two mice simultaneously and replicated the spatial memory deficits. Employing a single mouse at a time will eliminate the "social stress" component of the paradigm. This may produce the deficits, but we recommend that you consider this point in your experimental analyses.

2. The proposed restraint tube design can accommodate mice without operations or mice who have recovered from surgeries that are completely sealed with glue/sutures. Mice with implanted cannulae, optogenetic probes, electrodes, etc. will not fit in the tube as is. Mice with such implants can be unrestrained but tightly boxed off from each other or restrained in such a way that doesn't cover their head. The same idea applies to implants anywhere else on the body (e.g., catheters). 
3. The MAS paradigm can be performed once (Maras et al., 2014; Chen et al., 2016) or can be repeated over several days (Libovner et al., 2020).

4. MAS has been performed on C57BL/6 mice or mice with the C57BL/6 background in adolescents (Libovner et al., 2020) or adults (Maras et al, 2014; Chen et al., 2016). You may need to run pilot experiments on other genetic backgrounds or ages.

5. Stressed "peers" can be cage mates, mice from different cages, or a mixture. We would recommend stressing males and females separately.

B. Housing considerations

1. MAS should occur outside of the housing area and far enough such that noise will not be heard in the housing area. If sound attenuation is necessary, a sound barrier box can be constructed from mass-loaded vinyl (e.g., Mass Loaded Vinyl (MLV)-1lb.-Acoustic Barrier, amazon.com), foam blocks, and an appropriately sized cardboard box. In this case, a light source is placed in the box with the laboratory shaker and cage to conduct MAS.

2. Mice that are subjected to MAS should be housed separately from mice who are not because olfactory and other cues from the stressed mouse can be sensed by and in turn stress other mice (Brechbühl et al., 2013). Ideally an entire housing cage should be stressed together. If this is not feasible, mice should be separated from each other before being brought into the experiment room or days prior and remain separated. We have used mice that had been separated just prior to MAS, however this should be balanced with control mice that are also recently separated. If separations can be pre-planned, more days pre-separated before MAS and prior to any behavioral habituation sessions is preferable. Keep track of these housing situations for your experimental analyses.

3. In our experience, cage mates (both males and females) do not tend to become more aggressive in the days, weeks, or months following stress. Mice should be checked on regularly, however, to ensure no issues arise.

C. Behavioral testing considerations

1. MAS should not occur in a behavioral suite in which the same mice will be tested.

2. Many behavioral paradigms (e.g., object location memory, object recognition memory) involve extensive handling or habituation periods prior to measuring the behavioral outcome of interest. To observe the immediate effects of MAS on these measures, handling or habituation can be done leading up to MAS. For example, in Chen et al., 2016 mice were habituated to the testing apparatus for five to six days preceding MAS. Mice were then exposed to MAS on the day following the last habituation session then trained following a "rest period." To study long-term effects of MAS these procedures can occur before and/or following MAS as needed. Wellhandled mice will be easier to restrain and will thus require less "stressful" handling.

3. To study the immediate effects of MAS on behavioral tasks, tasks can be performed on the same day but should occur after a brief "rest period" post stress. Work from the Baram lab has shown 
that two hours after stress has been completed, corticosterone levels were similar between stress and control mice. Importantly, by that time point mice explored the environment and objects similarly to controls (Chen et al., 2016).

4. Several mice can be subjected to MAS simultaneously. If a consistent time period is desired between MAS and a behavioral task (or culling) that can only accommodate a few subjects at a time, consider staggering mice across the paradigm. Begin MAS as described with one or a few mice. At a later time point, additional mice can be restrained outside of the room or sound proofed container then quickly transported to the shaker. Once the desired MAS duration has elapsed for the initial mice, remove the tubes and un-restrain these mice outside of the room. Ensure each mouse is exposed to MAS for the desired duration before removing from the shaker and restraint.

\section{Acknowledgments}

We thank Hollis Rhodes for editorial insight and Aidan Pham for technical assistance. This work is funded by NS28912 and MH73136 to TZB, the Hewitt Foundation for Biomedical Research to JLB and TZB, and The Whitehall Foundation \#2018-12-09 to GL. This protocol was adapted from published work from the labs of TZB (Maras et al., 2014; Chen et al., 2016) and GL (Libovner et al., 2020).

\section{Competing interests}

The authors declare no conflict of interest.

\section{Ethics}

The above protocol is performed in accordance with the $\mathrm{NIH}$ guidelines on laboratory animal welfare and has been approved by UCl's Institutional Animal Care and Use Committee (IACUC): AUP-18183 valid December 2018 through December 2021 and AUP-17-145 valid September 2017 through September 2020.

\section{References}

1. Brechbühl, J., Moine, F., Klaey, M., Nenniger-Tosato, M., Hurni, N., Sporkert, F., Giroud, C. and Broillet, M. C. (2013). Mouse alarm pheromone shares structural similarity with predator scents. Proc Natl Acad Sci U S A 110(12): 4762-4767.

2. Brivio, P., Sbrini, G., Riva, M. A. and Calabrese, F. (2020). Acute stress induces cognitive improvement in the novel object recognition task by transiently modulating bdnf in the prefrontal cortex of male rats. Cell Mol Neurobiol. 
3. Burstein, O. and Doron, R. (2018). The unpredictable chronic mild stress protocol for inducing anhedonia in mice. $J$ Vis $\operatorname{Exp}(140)$.

4. Chen, Y., Molet, J., Lauterborn, J. C., Trieu, B. H., Bolton, J. L., Patterson, K. P., Gall, C. M., Lynch, G. and Baram, T. Z. (2016). Converging, synergistic actions of multiple stress hormones mediate enduring memory impairments after acute simultaneous stresses. J Neurosci 36(44): 11295-11307.

5. Kim, K. S. and Han, P. L. (2006). Optimization of chronic stress paradigms using anxiety- and depression-like behavioral parameters. J Neurosci Res 83(3): 497-507.

6. Libovner, Y., Fariborzi, M., Tabba, D., Ozgur, A., Jafar, T. and Lur, G. (2020). Repeated exposure to multiple concurrent stresses induce circuit specific loss of inputs to the posterior parietal cortex. J Neurosci 40(9): 1849-1861.

7. Lowe, S. R. and Galea, S. (2017). The mental health consequences of mass shootings. Trauma Violence Abuse 18(1): 62-82.

8. Maras, P. M., Molet, J., Chen, Y., Rice, C., Ji, S. G., Solodkin, A. and Baram, T. Z. (2014). Preferential loss of dorsal-hippocampus synapses underlies memory impairments provoked by short, multimodal stress. Mol Psychiatry 19(7): 811-822.

9. Monteiro, S., Roque, S., de Sá-Calçada, D., Sousa, N., Correia-Neves, M. and Cerqueira, J. J. (2015). An efficient chronic unpredictable stress protocol to induce stress-related responses in C57BL/6 mice. Front Psychiatry 6: 6.

10. Musazzi, L., Tornese, P., Sala, N. and Popoli, M. (2017). Acute or Chronic? A Stressful Question. Trends Neurosci 40(9): 525-535.

11. North, C. S., Smith, E. M. and Spitznagel, E. L. (1994). Posttraumatic stress disorder in survivors of a mass shooting. Am J Psychiatry 151(1): 82-88.

12. Novotney, A. (2018). What happens to the survivors. APA 49(8): 36.

13. Peay, D. N., Saribekyan, H. M., Parada, P. A., Hanson, E. M., Badaruddin, B. S., Judd, J. M., Donnay, M. E., Padilla-Garcia, D. and Conrad, C. D. (2020). Chronic unpredictable intermittent restraint stress disrupts spatial memory in male, but not female rats. Behav Brain Res 383: 112519.

14. Willner, P. (1997). Validity, reliability and utility of the chronic mild stress model of depression: a 10-year review and evaluation. Psychopharmacology (Berl) 134(4): 319-329.

15. Willner, P. (2017). The chronic mild stress (CMS) model of depression: History, evaluation and usage. Neurobiol Stress 6: 78-93. 